\title{
On barycentrically soft compacta
}

\author{
by
}

\author{
T. Radul (L'viv)
}

\begin{abstract}
It is shown that a barycentrically soft compactum is necessarily an absolute retract of weight $\leq \omega_{1}$. Since softness of a map is the mapping version of the property of a space to be an absolute retract, the above mentioned result can be considered as mapping version of the Ditor-Haydon Theorem stating that if $P(X)$ is an absolute retract then the compactum $X$ is of weight $\leq \omega_{1}[2]$.
\end{abstract}

All spaces considered are assumed to be compacta (compact Hausdorff spaces). For a compactum $X$ let $C(X)$ be the space of all real-valued continuous functions on $X$ metrized by sup-metric and let $P(X)$ be the space of all non-negative functionals $\mu: C(X) \rightarrow \mathbb{R}$ with norm 1 , equipped with the weak* topology.

Recall that the base of the weak* topology in $P(X)$ consists of the sets of the form

$O\left(\mu_{0}, f_{1}, \ldots, f_{n}, \varepsilon\right)=\left\{\mu \in P(X)|| \mu\left(f_{i}\right)-\mu_{0}\left(f_{i}\right) \mid<\varepsilon\right.$ for every $\left.1 \leq i \leq n\right\}$.

Let $E$ be a locally convex vector space. Then for any convex compact subset $K \subset E$ there exists a map $b=b_{K}: P(X) \rightarrow K$ which is called the barycentric map of probability measures. It is defined by $b(\mu)=\int x d \mu(x)$, where $x=\mathrm{id}_{E}$. The map $b_{K}$ is continuous [1]. It is not difficult to check that for $\mu=a_{1} \delta_{x_{1}}+\ldots+a_{n} \delta_{x_{n}}, a_{i} \in \mathbb{R}, x_{i} \in K$, we have $b_{K}(\mu)=a_{1} x_{1}+$ $\ldots+a_{n} x_{n}$, where $\delta_{x_{i}}$ denotes the Dirac measure supported by $x_{i}$.

A map $f: X \rightarrow Y$ is said to be (0-)soft if for any (0-dimensional) paracompact space $Z$, any closed subspace $A$ of $Z$ and maps $\Phi: A \rightarrow X$ and $\Psi: Z \rightarrow Y$ with $\Psi \mid A=f \circ \Phi$ there exists a map $G: Z \rightarrow X$ such that $G \mid A=\Phi$ and $\Psi=f \circ G$. This notion was introduced by E. Shchepin [9].

1991 Mathematics Subject Classification: 46E27, 54C10, 54C55.

Key words and phrases: barycentric map, soft map, probability measures.

The research was supported by State Committee for Science and Technology, Ukraine. 
A convex compactum $K$ is said to be barycentrically soft (open) if the barycentric map $b_{K}$ is soft (open). V. Fedorchuk [3] has given a criterion of barycentric openness of compacta which, in particular, implies that for every compactum $X$ the compactum $P(X)$ is barycentrically open. He has also shown in [4] that the product of a family of cardinality $\omega_{1}$ of barycentrically soft compacta is barycentrically soft and in the survey article [5] he has formulated the following questions concerning barycentric softness of compacta:

1) [5, Question 7.13] Is there a barycentrically soft compactum of weight $\geq \omega_{2}$ ?

It is worth noticing that the space $P(X)$ is not an absolute retract for any compactum of weight $\geq \omega_{2}[2]$. So we naturally obtain:

2) [5, Question 7.14] Is every barycentrically soft compactum an absolute retract?

3) [5, Question 7.15] Is every barycentrically open AR-compactum of weight $\omega_{1}$ barycentrically soft?

The author has answered the last question negatively: it is shown in [8] that the barycentric softness of a compactum of the form $P(X)$ is equivalent to the metrizability of $X$.

In this paper we answer questions 1) and 2) showing that every barycentrically soft compactum must be an absolute retract of weight $\leq \omega_{1}$.

In the sequel we shall need some definitions and results. Let

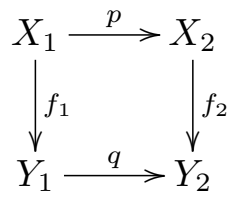

be a commutative diagram. The map $\chi: X_{1} \rightarrow X_{2} \times_{Y_{2}} Y_{1}=\{(x, y) \in$ $\left.X_{2} \times Y_{1} \mid f_{2}(x)=q(y)\right\}$ defined by $\chi(x)=\left(p(x), f_{1}(x)\right)$ is called the characteristic map of this diagram. The diagram is called bicommutative (respectively open, 0 -soft, soft) if the map $\chi$ is onto (respectively open, 0 -soft, soft).

It is shown in [6] that softness of a map with compact convex fibers is equivalent to its 0 -softness.

Let $\tau$ be an infinite cardinal number. A partially ordered set $\mathcal{A}$ is called $\tau$-complete if every subset of cardinality $\leq \tau$ has a least upper bound in $\mathcal{A}$. An inverse system of compacta and surjective bonding maps over a $\tau$ complete indexing set is called $\tau$-complete. A continuous $\tau$-complete system consisting of compacta of weight $\leq \tau$ is called a $\tau$-system.

The following theorem from [10] gives a characterization of 0 -soft maps: 
Theorem A. A map $f: X \rightarrow Y$ is 0-soft if and only if there exist $\omega$-systems $S_{X}$ and $S_{Y}$ with limits $X$ and $Y$ respectively and a morphism $\left\{f_{\alpha}\right\}: S_{X} \rightarrow S_{Y}$ with limit $f$ such that

1) $f_{\alpha}$ is 0-soft for every $\alpha$;

2) every limit square diagram is 0 -soft.

Lemma 1. Let $X$ and $Y$ be convex compacta and let $f: X \rightarrow Y$ be an affine non-open map. Then the diagram

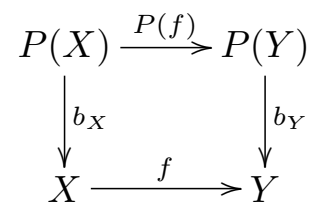

is non-open.

Proof. Since $f$ is non-open, the inverse map $f^{-1}: Y \rightarrow \exp X$ is not continuous. (By $\exp X$ we denote the hyperspace of $X$, i.e., the set of nonempty closed subsets of $X$ endowed with the Vietoris topology.)

Let $y_{0} \in Y$ be a discontinuity point of $f^{-1}$. Then there exist a net $\left\{y_{\alpha}\right\}_{\alpha \in \mathcal{A}}$ and a neighborhood $U$ of $f^{-1}\left(y_{0}\right)$ in $\exp X$ such that $y_{\alpha} \rightarrow y_{0}$ and $f^{-1}\left(y_{\alpha}\right) \notin U$ for every $\alpha \in \mathcal{A}$. We can assume that $f^{-1}\left(y_{\alpha}\right) \rightarrow A \in \exp X$. Since $f$ is a closed map, $A$ is a proper convex subset of $f^{-1}\left(y_{0}\right)$.

Choose points $x_{1} \in A$ and $x_{2} \in f^{-1}\left(y_{0}\right) \backslash A$. Replacing $x_{1}$ by $x_{1}^{\prime}=$ $(1-\lambda) x_{1}+\lambda x_{2}$, with $\lambda \in[0,1)$ chosen to be maximal subject to $x_{1}^{\prime} \in A$, we may assume that $\left(x_{1}+x_{2}\right) / 2 \notin A$. Since $A$ is convex, there exists an affine map $\psi$ that strictly separates the segment $\left[x_{2} ;\left(x_{1}+x_{2}\right) / 2\right]$ from $A$. We can assume that $\psi\left(\left[x_{2} ;\left(x_{1}+x_{2}\right) / 2\right]\right)<0$ and $\psi(A)>0$. Since $f^{-1}\left(y_{\alpha}\right) \rightarrow A$, there exists $\alpha_{0} \in \mathcal{A}$ such that $\psi \mid f^{-1}\left(y_{\alpha}\right)>0$ for every $\alpha>\alpha_{0}$. Consider a net $\left\{x_{\alpha}\right\}_{\alpha>\alpha_{0}}$ such that $x_{\alpha} \rightarrow x_{1}$ and the corresponding net in $P(X)$ defined by $\mu_{\alpha}=\left(\delta_{x_{2}}+\delta_{x_{\alpha}}\right) / 2$. Then $\chi\left(\mu_{\alpha}\right)=\left(\left(x_{2}+x_{\alpha}\right) / 2,\left(\delta_{y_{0}}+\delta_{y_{\alpha}}\right) / 2\right) \rightarrow$ $\left(\left(x_{2}+x_{1}\right) / 2, \delta_{y_{0}}\right)$. Now take the measure $\delta_{\left(x_{1}+x_{2}\right) / 2} \in \chi^{-1}\left(\left(x_{1}+x_{2}\right) / 2, \delta_{y_{0}}\right)$ and its neighborhood $O\left(\delta_{\left(x_{1}+x_{2}\right) / 2}, \phi, 1 / 4\right)$, where $\phi \in C(X)$ is a map such that $\phi\left(\left(x_{1}+x_{2}\right) / 2\right)=1$ and $\phi(x)=0$ for every $x \in X$ with $\psi(x) \geq 0$. Then $\mu(\phi)=1 / 2$ for every measure $\mu \in P(f)^{-1}\left(\left(\delta_{y_{0}}+\delta_{y_{\alpha}}\right) / 2\right)$, hence $\mu \notin$ $O\left(\delta_{\left(x_{1}+x_{2}\right) / 2}, \phi, 1 / 4\right)$ and the map $\chi$ is non-open. The lemma is proved.

A compactum $X$ is called openly generated if $X$ can be represented as the limit of an $\omega$-system with open bonding maps.

THEOREM 1. If a convex compactum $K$ is barycentrically soft, then $K$ is openly generated.

Proof. Present $K$ as the limit of an $\omega$-system $S_{K}=\left\{K_{\alpha}, p_{\alpha}, \mathcal{A}\right\}$, where the $K_{\alpha}$ are convex compacta and the bonding maps $p_{\alpha}$ are affine for every $\alpha \in \mathcal{A}$. 
If $b_{K}: P(K) \rightarrow K$ is soft, then, using the spectral theorem of $\mathrm{E}$. V. Shchepin [7, Theorem 3.12] and Theorem A, we deduce that there exists a closed cofinal subset $\mathcal{B} \subset \mathcal{A}$ such that for each $\alpha \in \mathcal{B}$ the diagram

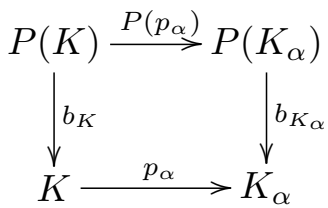

is 0 -soft and therefore open.

It follows from Lemma 1 that the map $p_{\alpha}$ is open for each $\alpha \in \mathcal{B}$. But since $K=\lim \left\{K_{\alpha}, p_{\alpha}, \mathcal{B}\right\}$, the compactum $K$ is openly generated. The theorem is proved.

THEOREM 2. Let $K$ be a barycentrically soft compactum. Then the weight of $K$ does not exceed $\omega_{1}$.

Proof. Let $K$ be a convex barycentrically open compactum of weight $\tau>\omega_{1}$. Suppose that the barycentric map is soft. Then there exist an embedding $i: P(K) \rightarrow I^{A} \times K$ and a retraction $r: I^{A} \times K \rightarrow P(K)$ such that the diagram

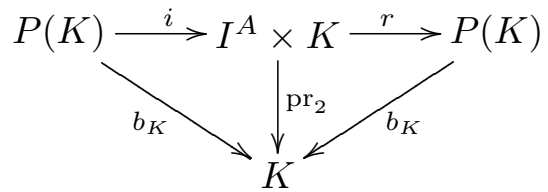

is commutative.

We can assume that the cardinality of $A$ is $\tau$. The compactum $K$ is assumed to be embedded in $I^{A}$. Present $K$ as the limit of an $\omega_{1}$-system $S=\left\{K_{\alpha}, p_{\alpha}, \mathcal{A}\right\}$, where the $K_{\alpha}$ are convex compacta, the $p_{\alpha}$ are affine maps for every $\alpha \in \mathcal{A}$, and $\mathcal{A}$ is the set of all subsets of cardinality $\leq \omega_{1}$ of $A$. Then $I^{A} \times K$ is the limit of the $\omega_{1}$-system $S^{\prime}=\left\{I^{B} \times K_{B}, q_{B}, B \in \mathcal{A}\right\}$ and $\mathrm{pr}_{K}$ is the limit of the morphisms determined by the family $\left\{\operatorname{pr}_{B} \mid B \in \mathcal{A}\right\}$. Since the map $b_{K}$ is embedded in $\operatorname{pr}_{K}$, we can assume that the restriction of the limit projections of the system $S^{\prime}$ onto $P(K)$ gives a morphism of inverse systems with the limit diagrams of the form

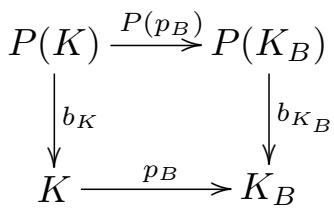

By Shchepin's Theorem (see [7]) there exists a cofinal closed subset $\mathcal{B} \subset$ $\mathcal{A}$ such that for every $B \in \mathcal{B}$ there exist an embedding $i_{B}: P\left(K_{B}\right) \rightarrow$ $I^{B} \times K_{B}$ and a retraction $r_{B}: I^{B} \times K_{B} \rightarrow P\left(K_{B}\right)$ for which the diagrams 


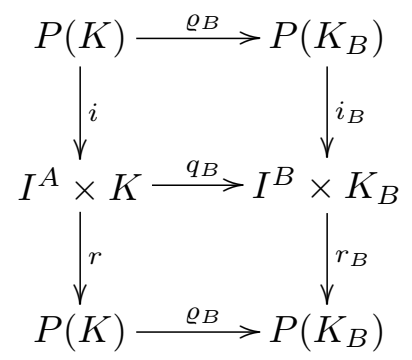

and

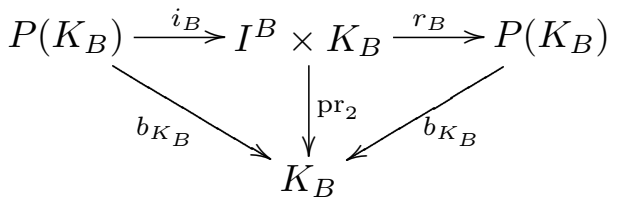

are commutative (here $\varrho_{B}$ denotes $P\left(p_{B}\right)$ and $q_{B}$ denotes the product of the corresponding projections from $I^{A}$ to $I^{B}$ and from $K$ to $K_{B}$ respectively).

Now choose sets $B, E \in \mathcal{B}$ such that $B \cap E=C \neq \emptyset, B \backslash C \neq \emptyset$ and $C \in \mathcal{B}$. We can do that by the method used in the proof of Theorem 3 of [2].

Let $T=\left\{\left(k_{1}, k_{2}\right) \in K_{B} \times K_{E} \mid p_{B}^{C}\left(k_{1}\right)=p_{E}^{C}\left(k_{2}\right)\right\}$, where $p_{B}^{C}: K_{B} \rightarrow K_{C}$ and $p_{E}^{C}: K_{E} \rightarrow K_{C}$ are the natural projections and $T_{P}=\left\{\left(\mu_{1}, \mu_{2}\right) \in\right.$ $\left.P\left(K_{B}\right) \times P\left(K_{E}\right) \mid P\left(p_{B}^{C}\right)\left(\mu_{1}\right)=P\left(p_{E}^{C}\right)\left(\mu_{2}\right)\right\}$.

For every $\left(\mu_{1}, \mu_{2}\right) \in T_{P}$ we have $i_{B}\left(\mu_{1}\right) \in I^{B} \times K_{B}, i_{E}\left(\mu_{2}\right) \in I^{E} \times K_{E}$, and for each $l \in C=B \cap E$ the $l$-coordinates of the points $i_{B}\left(\mu_{1}\right)$ and $i_{E}\left(\mu_{2}\right)$ are equal. Using this fact define $i_{T}: T_{P} \rightarrow I^{B \cup E} \times T$ by the conditions $s_{B} \circ i_{T}\left(\mu_{1}, \mu_{2}\right)=i_{B}\left(\mu_{1}\right)$ and $s_{E} \circ i_{T}\left(\mu_{1}, \mu_{2}\right)=i_{E}\left(\mu_{2}\right)$, where $s_{B}: I^{B \cup E} \times T \rightarrow I^{B} \times K_{B}$ and $s_{E}: I^{B \cup E} \times T \rightarrow I^{E} \times K_{E}$ are the natural projections.

Define $r_{T}: I^{B \cup E} \times T \rightarrow T_{P}$ by the conditions $r_{T} \circ s_{B}=r_{B}$ and $r_{T} \circ s_{E}=$ $r_{E}$. We can immediately check that $r_{T} \circ i_{T}=\mathrm{id}_{T_{P}}$.

The diagram

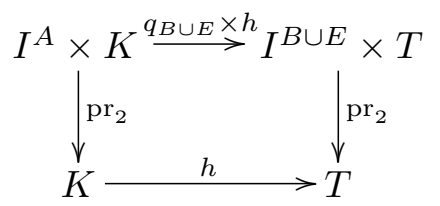

where $h=\left(p_{B}, p_{E}\right)$, is open.

But the diagram

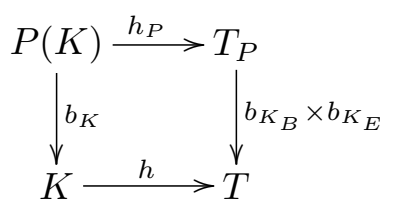


where $h_{P}=\left(P\left(p_{B}\right), P\left(p_{E}\right)\right)$, is a retract of $(1)$ and must be open as well. In order to finish the proof we have to show that (2) is non-open.

Consider the following three cases.

1) The map $h$ is open. We can assume that $p_{B}^{C}$ and $p_{E}^{C}$ are not homeomorphisms. Then there is a point $c_{1} \in K_{C}$ with non-singleton fiber with respect to $p_{B}^{C}$ and a point $c_{2} \in K_{C}$ with non-singleton fiber with respect to $p_{E}^{C}$. The fibers of $c=\left(c_{1}+c_{2}\right) / 2$ with respect to $p_{B}^{C}$ and $p_{E}^{C}$ are not single points. Now choose $b_{1}, b_{2} \in\left(p_{B}^{C}\right)^{-1}(c)$ and $e_{1}, e_{2} \in\left(p_{E}^{C}\right)^{-1}(c)$. We have $\left(b_{i}, e_{j}\right) \in T$ for every $i, j \in\{1,2\}$. Since $h$ is surjective, we can choose pairwise distinct points $k_{11}, k_{12}, k_{21}, k_{22}$ such that $p_{B}\left(k_{i 1}\right)=p_{B}\left(k_{i 2}\right)=b_{i}$ for $i \in\{1,2\}$ and $p_{E}\left(k_{1 j}\right)=p_{E}\left(k_{2 j}\right)=e_{j}$ for $j \in\{1,2\}$.

Let $\mu=\sum_{i, j=1}^{2} \frac{1}{4} \delta_{k_{i j}}$. Then

$$
\chi(\mu)=\left(\sum_{i, j=1}^{2} \frac{1}{4} k_{i j}, \frac{1}{2} \delta_{b_{1}}+\frac{1}{2} \delta_{b_{2}}, \frac{1}{2} \delta_{e_{1}}+\frac{1}{2} \delta_{e_{2}}\right)=(d, \nu, \eta) .
$$

Choose $k \in K$ such that $p_{C}(k) \neq c$. Let $b=p_{B}(k)$ and $e=p_{E}(k)$. Now put $b^{i}=\frac{1}{i+1} b+\frac{i}{i+1} b_{2}$ and $e^{i}=\frac{1}{i+1} e+\frac{i}{i+1} e_{2}$. Define the sequence of measures $\nu^{i} \in P\left(K_{B}\right)$ by $\nu^{i}=\frac{1}{2} \delta_{b^{i}}+\frac{1}{2} \delta_{b_{1}}$ and the sequence of measures $\eta^{i} \in P\left(K_{E}\right)$ by $\eta^{i}=\frac{1}{2} \delta_{e^{i}}+\frac{1}{2} \delta_{e_{1}}$.

It is obvious that $\nu^{i}$ converges to $\nu$ and $\eta^{i}$ converges to $\eta$. Since $h$ is open, we can choose a sequence $d_{i_{k}}$ in $K$ such that $d_{i_{k}}$ converges to $d$ and $p_{B}\left(d_{i_{k}}\right)=\frac{1}{2} b^{i_{k}}+\frac{1}{2} b_{1}, p_{E}\left(d_{i_{k}}\right)=\frac{1}{2} e^{i_{k}}+\frac{1}{2} e_{1}$. In $K \times_{T} T_{P}$ consider the sequence $\left(d_{i_{k}}, \nu^{i_{k}}, \eta^{i_{k}}\right)$ converging to $(d, \nu, \eta)$. Choose neighborhoods $V_{1}$ and $V_{2}$ of $e_{1}, e_{2}$ such that $\mathrm{cl} V_{1} \cap \mathrm{cl} V_{2}=\emptyset$ and neighborhoods $U_{1}, U_{2}$ of $b_{1}, b_{2}$ with the same property. Let $O_{i j}=p_{E}^{-1}\left(V_{j}\right) \cap p_{B}^{-1}\left(U_{i}\right)$. There exist functions $\phi_{i j} \in$ $C(K)$ such that $\phi_{i j}\left(k_{i j}\right)=1$ and $\phi_{i j} \mid\left(K \backslash O_{i j}\right)=0$ for every $i, j \in\{1,2\}$. We can assume that $b^{i_{k}} \in U_{2}$ and $e^{i_{k}} \in V_{2}$ for every $i_{k}$. Let $\mu^{\prime} \in \chi^{-1}\left(d_{i_{l}}, \nu^{i_{l}}, \eta^{i_{l}}\right)$ for some $i_{l}$. Then $P\left(p_{B}\right)\left(\mu^{\prime}\right)=\frac{1}{2} \delta_{b^{i_{l}}}+\frac{1}{2} \delta_{b_{1}}$, i.e., the measure $\mu^{\prime}$ takes on the value $1 / 2$ on the sets $p_{B}^{-1}\left(b^{i_{l}}\right)$ and $p_{B}^{-1}\left(b_{1}\right)$. Since $p_{B}^{C}\left(b^{i_{l}}\right) \neq k$, we have $p_{E}^{-1}\left(e_{1}\right) \cap p_{B}^{-1}\left(b^{i_{l}}\right)=\emptyset$. But $P\left(p_{E}\right)\left(\mu^{\prime}\right)=\frac{1}{2} \delta_{e^{i_{l}}}+\frac{1}{2} \delta_{e_{1}}$ and hence $\mu^{\prime}$ takes on the value $1 / 2$ on $p_{E}^{-1}\left(e_{1}\right) \cap p_{B}^{-1}\left(b_{1}\right) \subset O_{11}$. Reasoning similarly, we can prove that $\mu^{\prime}$ takes on the value $1 / 2$ on $O_{22}$. But then $\mu^{\prime}\left(O_{12}\right)=0$ and hence $\mu^{\prime} \notin O\left(\mu, \phi_{i j}, 1 / 8\right)$. We have shown that the diagram (2) is non-open whenever the map $h$ is open.

2) Let $h$ be non-open but surjective. In this case the proof is analogous to that of Lemma 1.

3) Let $h$ be non-surjective. We show that so is $\chi$. Let $\left(e_{1}, b_{1}\right) \in T$ and $\left(e_{1}, b_{1}\right) \notin h(K)$. Since $p_{B}$ and $p_{E}$ are surjective, we can choose $k_{B} \in p_{B}^{-1}\left(b_{1}\right)$ and $k_{E} \in p_{E}^{-1}\left(e_{1}\right)$. Let $p_{E}\left(k_{B}\right)=e_{2}$ and $p_{B}\left(k_{E}\right)=b_{2}$. Then $\left(e_{1}, b_{2}\right) \in$ $h(K),\left(e_{2}, b_{1}\right) \in h(K)$, and $\left(e_{2}, b_{2}\right) \in T$. But then $\frac{1}{2}\left(e_{1}, b_{1}\right)+\frac{1}{2}\left(e_{2}, b_{2}\right)=$ 
$\frac{1}{2}\left(e_{2}, b_{1}\right)+\frac{1}{2}\left(e_{1}, b_{2}\right) \in h(K)$. We can assume that $k=\frac{1}{4}\left(e_{2}, b_{2}\right)+\frac{3}{4}\left(e_{1}, b_{1}\right)$ $\in h(K)$. Define $\nu=\frac{1}{4} \delta_{b_{2}}+\frac{3}{4} \delta_{b_{1}}, \eta=\frac{1}{4} \delta_{e_{2}}+\frac{3}{4} \delta_{e_{1}}$ and choose $z \in h^{-1}(k)$. Then $(z, \nu, \eta) \in K \times_{T} T_{P}$. But since $p_{B}^{-1}\left(b_{1}\right) \cap p_{E}^{-1}\left(e_{1}\right)=\emptyset$, we have $\chi^{-1}(z, \nu, \eta)=\emptyset$.

Thus the diagram (2) is non-open.

Since the properties of being an AR-compactum and of being an $\mathrm{AE}(0)$ compactum coincide in the class of convex compacta and each openly generated compactum of weight $\leq \omega_{1}$ is an $\mathrm{AE}(0)$-compactum, the following theorem is an immediate consequence of Theorems 1 and 2 .

TheOREM 3. A barycentrically soft compactum is necessarily an ARcompactum of weight $\leq \omega_{1}$.

The author would like to express his sincere thanks to V. Fedorchuk for drawing his attention to this problem and to M. Zarichnyu for valuable discussions.

\section{References}

[1] N. Bourbaki, Intégration, Hermann, Paris, 1963.

[2] S. Ditor and R. Haydon, On absolute retracts, $P(S)$ and complemented subspaces of $C\left(D^{\omega_{1}}\right)$, Studia Math. 56 (1976), 243-251.

[3] V. V.Fedorchuk, On the barycentric map of probability measures, Vestnik Moskov. Univ. Ser. 1, 1992 (1), 42-47 (in Russian).

[4] -, On barycentrically open bicompacta, Sibirsk. Mat. Zh. 33 (6) (1992), 205-210 (in Russian).

[5] —, Probability measures in topology, Uspekhi Mat. Nauk 46 (1) (1991), 41-80 (in Russian).

[6] -, On softness of maps of subsets in locally convex spaces, ibid. 45 (2) (1990), 221-222 (in Russian).

[7] V. V. Fedorchuk and V. V. Filippov, General Topology. Fundamental Constructions, Izdat. Moskov. Univ., Moscow, 1988 (in Russian).

[8] T. Radul, On the barycentric map of probability measures, Vestnik Moskov. Univ. Ser. 1, 1994 (1), 3-6 (in Russian).

[9] E. V. Shchepin, Functors and uncountable powers of compacta, Uspekhi Mat. Nauk 36 (3) (1981), 3-62 (in Russian).

[10] M. M. Zarichny $\breve{1}$, Absolute extensors and the geometry of multiplication of monads in the category of compacta, Mat. Sb. 182 (1991), 1261-1280 (in Russian).

L'VIV STATE UNIVERSITY

DEPARTMENT OF MATHEMATICS

UNIVERSITETSKA ST., 1

290602 L'VIV, UKRAINE 